\title{
Femtosecond Laser Structuring of As2S3 Glass for Erasable and Permanent Optical Memory
}

Saulius Juodkazis ${ }^{1}$, Andrei V. Rode ${ }^{2}$, Toshiaki Kondo ${ }^{1}$, Hiroaki Misawa ${ }^{1}$, Marek Samoc ${ }^{2}$, and Barry Luther-Davies ${ }^{2}$

${ }^{1}$ Hokkaido University, Sapporo, 001-0021, Japan

${ }^{2}$ The Australian National University, Canberra, 0200, Australia

\begin{abstract}
The nonlinear absorption coefficient of $\mathrm{As}_{2} \mathrm{~S}_{3}$ glass has been measured to be $2.0 \mathrm{~cm} / \mathrm{GW}$ for femtosecond pulses at $800 \mathrm{~nm}$. Femtosecond laser structuring via two photon absorption in bulk $\mathrm{As}_{2} \mathrm{~S}_{3}$ glass by erasable and permanent photo-darkening is demonstrated using both holographic and direct multi-beam laser writing.
\end{abstract}

\section{INTRODUCTION}

Chalcogenide glasses are nonlinear optical materials with considerable potential for alloptical switching at the IR telecommunications wavelengths [1-4]. A general characteristic of chalcogenides is their susceptibility to photo-darkening which can result in a refractive index change of $\Delta n \sim 10^{-2}$ that can be used for creating thermally re-writable optical memories and for applications in micro-photonics. This work explores the use of nonlinear absorption of femtosecond laser pulses to achieve permanent/erasable 3D optical data storage $[5,6]$ in a photosensitive chalcogenide glass- a process which has the potential to significantly boost memory storage capacity.

\section{EXPERIMENT}

Ultrafast (sub-ps) 800-nm laser pulses were focused into a block of transparent $\mathrm{As}_{2} \mathrm{~S}_{3}$ glass to record a three-dimensional (3D) pattern via photo-darkening. The formation of "memory bits" involves two-photon absorption and can be achieved using relatively low energy laser pulses. This provides the opportunity for fast parallel writing of multi-bit patterns using a single laser pulse.

Commercial $\mathrm{As}_{2} \mathrm{~S}_{3}$ glass (Amorphous Materials) with a melting temperature of $310^{\circ} \mathrm{C}$ was used in this study. Amplified femtosecond laser pulses (wavelength $800 \mathrm{~nm}$, pulse duration 150fs) were obtained from a Spectra Physics Hurricane laser operating at $1 \mathrm{kHz}$ repetition rate. Holographic recording was realized by 4- and 5-beam interference using a diffractive optical element (DOE) (see, ref. $[7,8]$ for details) and focusing with a $N A=0.75$ objective lens. 


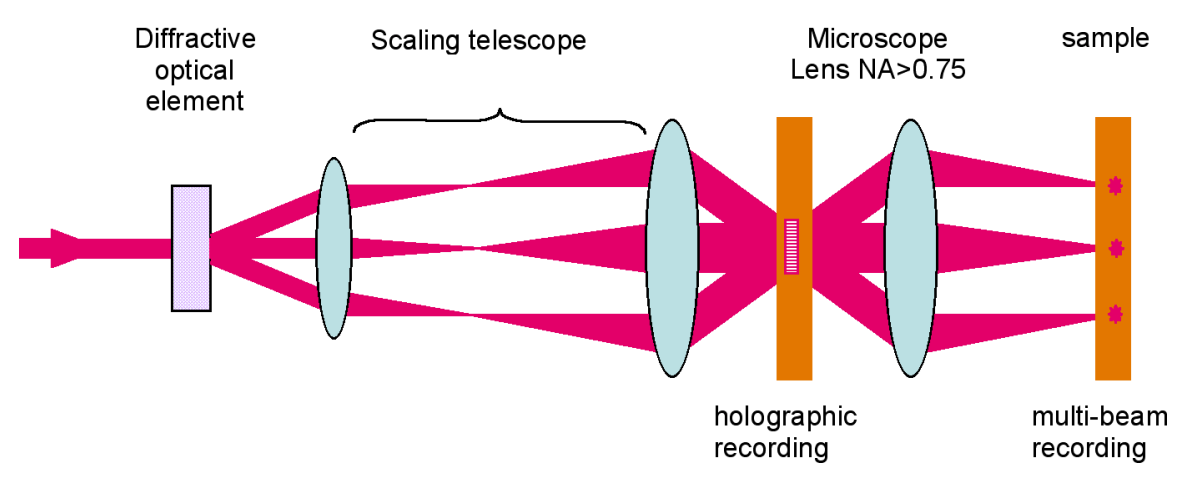

Figure 1. Schematic setup based on diffractive optical element (DOE) for holographic and direct multi-beam recording.

For direct laser writing, the holographic setup was modified by simply adding a second lens (Fig. 1). In this geometry a DOE generating 31 beamlets (G1022A) in a single line was utilized and the sample was translated normal to the line there by recording 31 lines in one scan. The scan speed was $0.1 \mathrm{~mm} / \mathrm{s}$, which corresponded to $20 \mathrm{~nm}$ between successive pulses. The whole sample was then translated laterally by half the pattern width and re-exposed. By this process the line exposed to the most off-center beamlet (the weakest) was subsequently overwritten with the strongest $0^{\text {th }}$ beamlet during the second scan resulting in the formation of a uniform pattern over a comparatively large $\left(\sim 1 \mathrm{~cm}^{2}\right)$ area.

The nonlinear absorption of $\mathrm{As}_{2} \mathrm{~S}_{3}$ was measured between $650 \mathrm{~nm}$ and $1200 \mathrm{~nm}$ using the $\mathrm{z}-$ scan technique with a TOPAS (Light Conversion) optical parametric generator pumped with a femtosecond Clark-MXR CPA2001 laser. The beam from the TOPAZ was focused into a sample $2.74 \mathrm{~mm}$ thick using an $f=150 \mathrm{~mm}$ lens and in some conditions spatially filtered with a pair of apertures to give a truncated Airy disk pattern as described in ref. [9]. Prior to running Zscans on $\mathrm{As}_{2} \mathrm{~S}_{3}$ the light intensity was calibrated using closed aperture Z-scans on fused silica plates 1-3 mm thick for which the nonlinearity is known to be $n_{2}=3 \times 10^{-16} \mathrm{~cm}^{2} / \mathrm{W}$.

Figure 2 shows a set of $Z$-scans recorded at $820 \mathrm{~nm}$ for a range of peak intensities. The curves were fitted by numerical integration of Sheikh-Bahae equations [10] to determine the non-linear absorption parameters. As an example, Fig. 2(b) shows the fit for the lowest intensity from Fig. 2(a) $\left(0.839 \mathrm{GW} / \mathrm{cm}^{2}\right)$ which resulted in the imaginary part of the nonlinear phase shift $\operatorname{Im}(\Delta \varphi)=0.30 \mathrm{rad}$. A large number of data points were recorded at various intensities and from these it was determined that $\operatorname{Im}(\Delta \varphi) / I=0.547 \mathrm{rad} \mathrm{cm}^{2} / \mathrm{GW}$ at $820 \mathrm{~nm}$. Taking into account the sample thickness one calculates from

$$
\operatorname{Im}\left(n_{2}\right)=\frac{\lambda}{2 \pi L} \frac{\operatorname{Im}(\Delta \varphi)}{I}
$$

that $\operatorname{Im}\left(n_{2}\right)=3.1 \times 10^{-14} \mathrm{~cm}^{2} / \mathrm{W}$ and, from

$$
\beta=\frac{2 \pi}{\lambda} \operatorname{Im}\left(n_{2}\right)
$$

the two-photon absorption coefficient of $\beta=2.0 \pm 0.2 \mathrm{~cm} / \mathrm{GW}$. Since the two-photon absorption cross section can be expressed as $\sigma_{2}=\beta \hbar \omega / N$ and the molecular density of $\mathrm{As}_{2} \mathrm{~S}_{3}$ molecules is $N=N_{0} \rho / M \cong 7.8 \times 10^{21} \mathrm{~cm}^{-3}$, we obtain $\sigma_{2}=6.2 \times 10^{-50} \mathrm{~cm}^{4} \mathrm{~s}$ or 6.2 Goeppert-Mayers (GM) for a formally stoichiometric molecule. 
The wavelength dependence of the two-photon absorption coefficient for $\mathrm{As}_{2} \mathrm{~S}_{3}$ obtained from Z-scans at different wavelengths is shown in Fig. 2. The scatter in the results primarily arises from uncertainties in determining the intensity in the samples and the possibility that at high intensity the approximation used in the Sheikh-Bahae theory [10] was broken and higherorder absorption could affect the measurements. The large amount of data collected at $820 \mathrm{~nm}-$ close to the wavelength used in the experiments on two-photon induced photo-darkening - led to better accuracy at this wavelength.
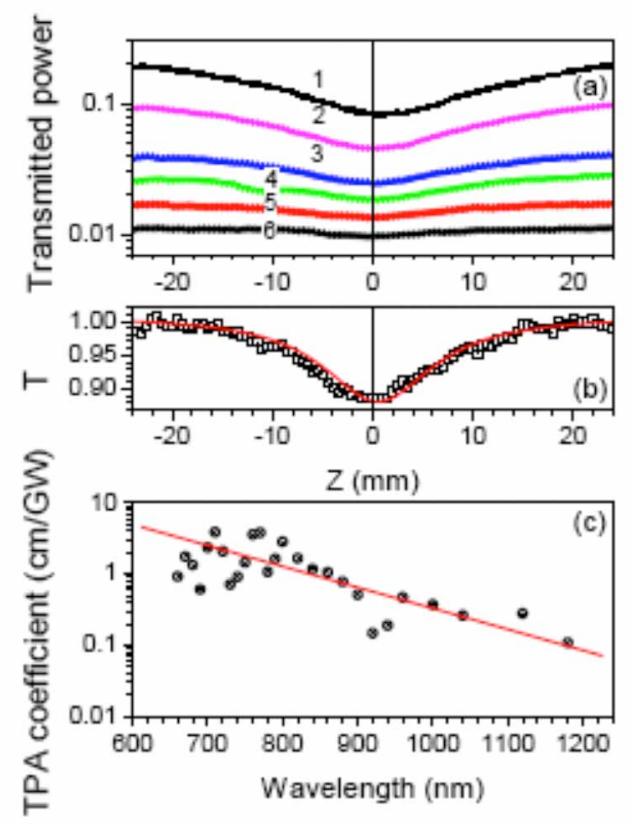

Figure 2. Z-scan measurements: (a) a set of open aperture Z-scans at $820 \mathrm{~nm}$ for the following light intensities: $14.5,7.2,3.0,2.1,1.22$ and $0.839 \mathrm{GW} / \mathrm{cm}^{2}$; (b) numerical fit of transmission, $T$, for the intensity of $0.839 \mathrm{GW} / \mathrm{cm}^{2}$; (c) Wavelength dependence of the two-photon absorption coefficient; the straight line is an exponential fit given as an eye guide.

The laser intensity thresholds for erasable and permanent two-photon photo-modification were measured and compared with those for conventional single-photon direct laser writing using a cw-laser [2-4]. The single pulse threshold for erasable photo-darkening for a $800 \mathrm{~nm} / 180$ fs pulse was determined by focused beam direct laser writing to be $5.7 \pm 0.5 \mathrm{GW} / \mathrm{cm}^{2}$ $\left(<1 \mathrm{~mJ} / \mathrm{cm}^{2}\right)$ for three-dimensional patterns written approximately $20 \mu \mathrm{m}$ beneath the surface using the $N A=0.75$ objective lens. Erasing was achieved by annealing the sample at $150 \pm 15^{\circ} \mathrm{C}$ for $2 \mathrm{~h}$. The threshold was determined using a beam with minimized spherical aberration by changing the divergence of incoming beam to achieve photodarkening with the smallest crosssection at the smallest pulse energy at a fixed $20 \mu \mathrm{m}$ depth beneath the target surface. Near the threshold irradiance lines of exposures using single pulse separated by $400 \mathrm{~nm}$ were created and this allowed the onset of photo-modification to be clearly identified by in-situ observation. The dielectric breakdown threshold recognizable by a plasma spark occurred at approximately $<0.18$ $\mathrm{J} / \mathrm{cm}^{2} /$ pulse $\left(8.2 \times 10^{11} \mathrm{~W} / \mathrm{cm}^{2} /\right.$ pulse irradiance $)$. Such fluences are obtainable from standard fsoscillator [5], however, we used an amplified laser at $1 \mathrm{kHz}$ repetition rate and lower average power to avoid the heat accumulation present in experiments with $\mathrm{MHz}$ oscillators. The single pulse threshold fluence at $1 \mathrm{kHz}$ was significantly $\left(>10^{2}\right.$ times $)$ smaller than that observed in 
experiments with fs-/MHz-lasers [1] but the irradiance was larger. Permanent (thermally unerasable) photodarkening was observed at approximately $10^{-2} \mathrm{~J} / \mathrm{cm}^{2}$ using the same measurement procedure. It should be noted that the data on photordarkening discussed in what follows were strongly affected by spherical aberration and also involved multiple pulses, hence, the thresholds determined above cannot be directly compared with fluence/irradiance used in the following experiments.

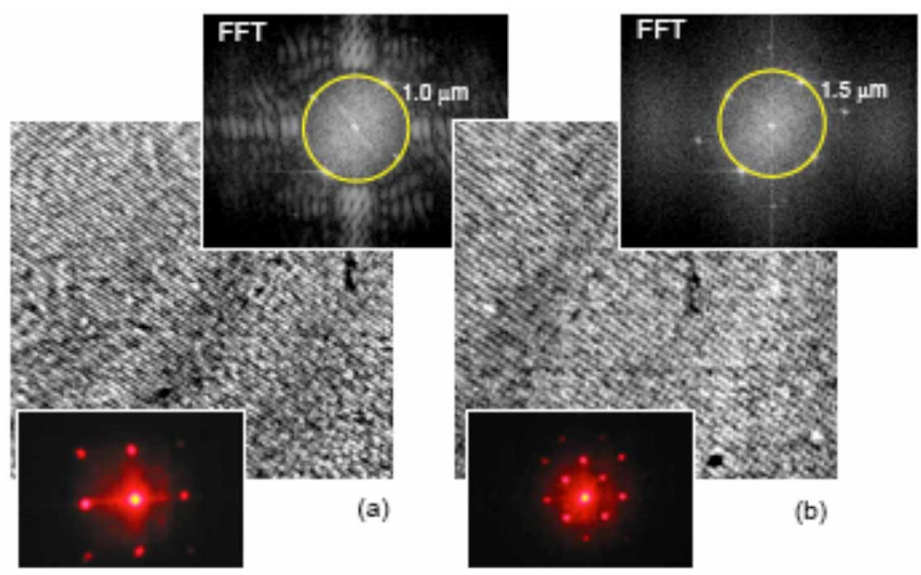

Figure 3. Optical transmission images of four (a) and five (b) beams holograms and their Fourier transform images: numerical (upper inset) and by diffraction readout by $632 \mathrm{~nm}$ laser light (lower inset). Theoretical period was $1.02 \mathrm{~mm}$ (4-beams) and $1.45 \mathrm{~mm}$ (5-beams). Holograms were recorded by a 5 min exposure to the $3 \mathrm{~mJ} /$ pulse (at $1 \mathrm{kHz}$ repetition rate) pulses of $800 \mathrm{~nm}$ wavelength and $150 \mathrm{fs}$ duration. Area of pattern $0.7 \mathrm{~mm}$ at $20 \mathrm{~mm}$ depth; angle of focusing was $33.6^{\circ}$.

Four- and five-beam holograms were formed inside $\mathrm{As}_{2} \mathrm{~S}_{3}$ glass to check a possibility of recording a $3 \mathrm{D}$ hologram by photodarkening. The light intensity distribution $I(r)$ creating the hologram can be simulated by interference of plane waves as:

$$
I(r)=\sum_{n, m} \vec{E}_{n} \exp \left[-i\left(\vec{k}_{n} \vec{r}+\delta_{n}\right)\right] * \vec{E}_{m}^{*} \exp \left[i\left(\vec{k}_{m} \vec{r}+\delta_{m}\right)\right]
$$

where $E$ is the $E$-field vector, * designates a complex conjugate), $r$ is the coordinate vector, $k$ is the wave vector, and $n=m$ represents the number of interfering beams. The phases of beams are given by $\delta_{n, m}=0$. Control of the phases provides a method for fine tuning of the interference patterns. Calculations were carried out for s-polarization (perpendicular to the plane of incidence), hence there was no depolarization present, i.e., the longitudinal component $E_{z}=0$.

The resulting optical images of the pattern of photodarkening formed holographically are shown in Fig. 3. These images were digitized and Fourier transformed numerically. The resulting calculated diffraction patterns are shown in the inset with the dominant (smallest) period identified and this corresponded well tot hetheoretically determined value appropriate for the focusing conditions employed in the experiment. The diffraction pattern of He-Ne beam (see lower inset) was also recorded and shows the same periodicity. This implies, however, that the actual pattern recorded by4-/5-beams inside the glass was planar rather than three-dimensional as expected, i.e. the optical diffraction was the same as calculated from $2 \mathrm{D}$ optical transmission image. 
The actual axial length of the 4-/5-beams pattern was evaluated by measuring the angular dependence of the diffraction efficiency from a grating recorded by two beam interference. The first minimum of the diffraction was observed at $29^{\circ}$ at largest saturated value of photodarkening. The highest photodarkening value of $\Delta n=0.06$ (at $633 \mathrm{~nm}$ ) was obtained in $\mathrm{As}_{2} \mathrm{~S}_{3}[3,4]$. Then, the $29^{\circ}$ corresponds to $10 \mu \mathrm{m}$ axial length. At the third of the saturated exposure, $\Delta n=0.02 \pm 0.005$, the position of the first minimum of th eangular dependence of diffraction efficiency was $18^{\circ}$, which corresponds to the axial extent (thickness) of a photodarkened region $d=20 \pm 5 \mu \mathrm{m}$. It is noteworthy, that in order to avoid total internal reflection and to measure the transmitted first-order diffraction, the cover glass was attached onto the backside of As2S3 sample using immersion oil $(n=1.515$ at $633 \mathrm{~nm})$. This expanded the angular range of the measurable diffraction up to $30^{\circ}$.

The depth of the pattern formed by 4-/5-beams inside the $\mathrm{As}_{2} \mathrm{~S}_{3}$ sample was considerably smaller than would be expected from the focusing conditions. The effective numerical aperture of beams writing the pattern was 5-6times smaller than that of the objective lens, $N A=0.75$, since the diameter of the beamlets was approximately $1 \mathrm{~mm}$ (the entrance aperture of the objective lens was $5 \mathrm{~mm}$ ). Hence, the axial extent of the waist (the depth of focus), was expected to be approximately $50 \mu \mathrm{m}$. The experimentally measured axial extent of the photodarkened region of only $d=20 \pm 5 \mu \mathrm{m}$, was also confirmed by direct optical imaging. The discrepancy between the theoretical and experimental length of the pattern can be explained by the photodarkening itself, which creates an array of micro-lenses which modify the distribution of the light preventing hologram formation deeper into the sample. This is a fundamental constraint of 3D structuring: once the refractive index is changed, the focusing is altered for the regions further along the light propagation. In the case of holographic recording, the 3D intensity distribution was distorted when photo-darkening occurred at locations of the highest intensity. The 3D structures still could be recorded by translating sample axially in steps equal to the axial period along the direction of light propagation.

A simple addition of one lens allowed us to change the holographic recording setup into a multi-beam direct laser writing tool. Such extension of the holographic recording is equivalent to the more efficient photo-structuring by a lens array introduced recently [11]. The effective numerical aperture of the objective lens for multi-beam recording becomes smaller by the ratio of the beamlet diameter to that of entrance aperture of the objective lens. The pattern of lines written by slow scanning using the lateral shift described earlier has proved to create extended gratings with high structural quality. The pattern could not be erased by a $150^{\circ} \mathrm{C} / 2 \mathrm{~h}$ annealing procedure (no changes of optical density were observed). This method can be used for waveguide formation as well.

\section{CONCLUSIONS}

Rewritable and permanent photo-structuring of $\mathrm{As}_{2} \mathrm{~S}_{3}$ glass by femtosecond laser pulses has been demonstrated. Both methods can be applied for efficient large-area $(<\mathrm{mm})$ pattern formation. The two-photon absorption coefficient was measured for $\mathrm{As}_{2} \mathrm{~S}_{3}$ chalcogenide glass in a wide spectral range from 650 to $1200 \mathrm{~nm}$. The two-photon absorption cross-section was found to be $6.2 \pm 0.5 \mathrm{GM}$ around $800 \mathrm{~nm}$ wavelength. The threshold laser intensities for erasable and permanent photodarkening for single $800 \mathrm{~nm} / 180 \mathrm{fs}$ pulses were $1 \mathrm{~mJ} / \mathrm{cm}^{2}$ per pulse and 10 $\mathrm{mJ} / \mathrm{cm}^{2}$ per pulse (dielectric breakdown at $<0.18 \mathrm{~J} / \mathrm{cm}^{2}$ per pulse) correspondingly in $\mathrm{As}_{2} \mathrm{~S}_{3}$. The 
results demonstrate that the use of chalcogenide media modified by ultrafast laser pulses through a photodarkening process has a great potential for fast 3D laser writing of erasable and permanent optical memory approaching a TBit $/ \mathrm{cm}^{3}$ memory density domain (estimated from a single bit volume $\lambda \times \lambda \times 2 \lambda$ at focus with objective lens of $N A=0.75$ ).

This presentation is a concise version of the paper published in [12].

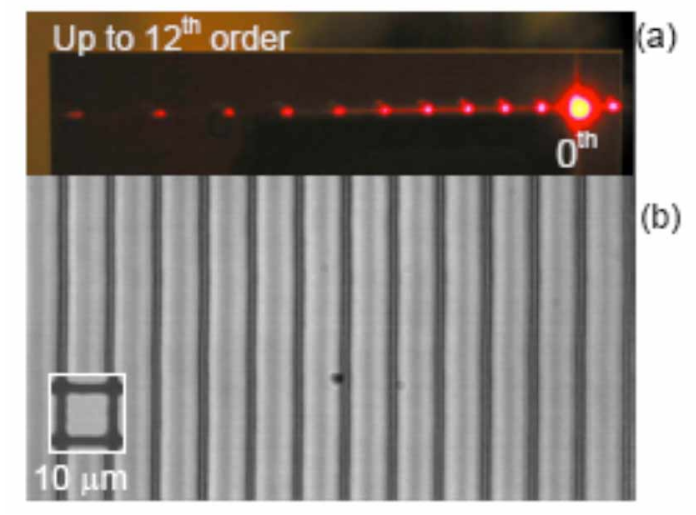

Figure 4. (a) Diffraction pattern of $\mathrm{HeNe}$ laser beam on the grating recorded by direct multibeam (with 31 inline beams) laser writing; (b) optical image of the grating in $\mathrm{As}_{2} \mathrm{~S}_{3}$ glass.

\section{ACKNOWLEDGMENTS}

The support of the Australian Research Council through its Federation Fellow and Discovery programs is gratefully acknowledged.

\section{REFERENCES}

1. A. Zoubir, M. Richardson, C. Rivero, A. Schulte, C. Lopez, K. Richardson, N. Ho, and R. Valee, Opt. Lett. 29, $748-750$ (2004).

2. J.-F. Viens, C. Meneghini, A. Villeneuve, T. Galstian, E. Knystautas, M. Duguay, K. Richardson, and T. Cardinal, IEEE J. of Lightwave Technol. 17, 1184 - 1191 (1999).

3. A. Zakery, Y. Ruan, A. V. Rode, M. Samoc, and B. Luther-Davies, J. Opt. Soc. Am. B 20, 1844 1852 (2003).

4. A. V. Rode, A. Zakery, M. Samoc, R. B. Charters, E. G. Gamaly, and B. Luther-Davies, Appl. Surf. Sci. 197-198, 481 - 485 (2002).

5. C. B. Schaffer, J. Garcia, and E. Mazur, Appl. Phys. A 76, 351 - 354 (2003).

6. S. Juodkazis, A. V. Rode, E. G. Gamaly, S. Matsuo, and H. Misawa, Appl. Phys. B 77, 361-368 (2003).

7. T. Kondo, S. Matsuo, S. Juodkazis, and H. Misawa, Appl. Phys. Lett. 79, 725-727 (2001).

8. T. Kondo, S. Matsuo, S. Juodkazis, V. Mizeikis, and H. Misawa, J. Photopolym. Sci. Technol. 16, 427-432 (2003).

9. B. K. Rhee, J. S. Byun, and E. W. Van Stryland, J. Opt. Soc. Am. B 13, 2720 - 2723 (1996).

10. M. Sheikh-Bahae, A. A. Said, T. Wei, D. J. Hagan, and E. W. Van Stryland, IEEE J. Quantum Electron. 26, 760-769 (1990).

11. S. Matsuo, S. Juodkazis, and H. Misawa, Appl. Phys. A 80, 683 - 685 (2004).

12. S. Juodkazis, T. Kondo, H. Misawa, A. Rode, M. Samoc, B. Luther-Davies, Optics Express, 14, 7751-7756 (2006). 